\title{
Essais
}

ESSAIS

Revue interdisciplinaire d'Humanités

$14 \mid 2018$

Plurilinguismes en construction

\section{Les marques transcodiques dans les disciplines non linguistiques}

Comment les enseignants gèrent-ils l'apparition des langues premières chez les élèves allophones scolarisés au collège?

Elisabeth Faupin

\section{(2) OpenEdition}

Journals

Édition électronique

URL : http://journals.openedition.org/essais/315

DOI : 10.4000/essais.315

ISSN : 2276-0970

Éditeur

École doctorale Montaigne Humanités

Édition imprimée

Date de publication : 1 juin 2018

Pagination : 51-65

ISBN : 979-10-97024-06-2

ISSN : 2417-4211

Référence électronique

Elisabeth Faupin, «Les marques transcodiques dans les disciplines non linguistiques », Essais [En ligne], 14 | 2018, mis en ligne le 01 décembre 2019, consulté le 12 décembre 2019. URL : http:// journals.openedition.org/essais/315; DOI : 10.4000/essais.315 


\section{Les marques transcodiques dans les disciplines non linguistiques Comment les enseignants gèrent-ils l'appa- rition des langues premières chez les élèves allophones scolarisés au collège?}

\section{Elisabeth Faupin}

Avec les mouvements de populations qui s'accélèrent sur l'ensemble de la planète, chaque territoire se trouve peuplé par des individus aux origines et aux profils variés. Les motifs de déplacements sont multiples (opportunité professionnelle, conflits armés, expérience personnelle, départ précipité ou mûrement réfléchi, etc.) et ils engendrent évidemment des situations diverses. Ce sont souvent des familles entières qui sont amenées à vivre sur un nouveau territoire, dans une nouvelle langue et selon des mœurs différentes. Dans nos sociétés privilégiées, les expériences volontaires à l'étranger sont vues comme une opportunité, tant au niveau professionnel pour les adultes que pour la formation linguistique et culturelle des enfants. Cette valorisation de l'autre langue/culture comme bagage culturel ne concerne pourtant pas au même degré tous les territoires et toutes les langues. Notre ethnocentrisme, qu'il soit volontaire ou non, établit des hiérarchies dans la reconnaissance des langues et des cultures.

L'Éducation nationale, qui se charge d'accueillir et de former les élèves allophones nouvellement arrivés en France (EANA) n'échappe pas à cette problématique. Des élèves non ou partiellement francophones à leur arrivée en France doivent être scolarisés en français le plus rapidement possible pour poursuivre leurs études dans les meilleures conditions. Ces élèves sont inscrits dans les premier et second degrés et de nombreux paramètres entrent en jeu dans leur adaptation à l'école en français et leurs chances de réussir (âge, scolarité antérieure, projet familial dans le nouveau pays, etc.).

C'est dans cette perspective que nous avons centré notre recherche sur le cas des élèves allophones scolarisés depuis moins de deux ans au collège en France. Aujourd'hui, de nombreux établissements du second degré ont mis en place une structure d'accueil qui permet aux élèves allophones à la fois de suivre des cours de français avec un enseignant spécialisé en français langue 
seconde/langue de scolarisation ${ }^{1}$ et d'être inscrits en parallèle dans une classe ordinaire (avec des élèves francophones) qui correspond à leur âge ${ }^{2}$.

La question de la formation des élèves allophones en français se pose depuis plusieurs décennies et l'absence de matériel pédagogique adéquat a longtemps laissé les enseignants dans l'isolement. Plusieurs auteurs ont publié des manuels consacrés au français langue seconde en France 3 . Par ailleurs, des chercheurs comme Nathalie Auger, Fatima Chnane-Davin, Christine Hélot, Catherine Klein, Danièle Moore et Cécile Sabatier ${ }^{4}$ ont montré que ces élèves, qui doivent acquérir le français, n'arrivent pas en France vierges de tout savoir. Ils possèdent une langue et une culture, parfois plusieurs. Selon leur pays d'origine, leur langue première de scolarisation a pu être valorisée comme langue d'expression orale et écrite, comme langue d'acquisition de savoirs savants ; arrivés au collège en France, l'objectif prioritaire devient la maîtrise de la langue française. Les élèves peuvent valoriser leurs connaissances en mathématiques, par exemple, à partir du moment où leur niveau en français le leur permet, mais les langues maternelles et/ou les langues de première scolarisation font rarement partie du processus d'apprentissage. On ne s'appuie pas sur les langues connues des élèves pour poursuivre leur formation, créant ainsi une rupture alors que la scolarisation en France de ces élèves pourrait être vue comme une étape dans un projet plus général de formation d'un adulte en devenir.

C'est dans cette perspective que notre recherche sur les interactions orales en situation didactique au niveau du collège nous a amenée à observer les prises de parole des EANA dans les classes afin d'analyser le contexte des apparitions de langues autres que la langue de scolarisation (ici le français) durant les cours ainsi que l'accueil réservé par les enseignants à ces interventions allogènes.

1 Note de service n ${ }^{\circ} 2004-175$ du 19 octobre 2004. Attribution aux personnels enseignants des premiers et second degrés relevant du MEN d'une certification complémentaire dans certains secteurs disciplinaires.

2 Circulaire $\mathrm{n}^{\circ}$ 2012-141 du 2 octobre 2012. Organisation de la scolarité des élèves allophones nouvellement arrivés. $\mathrm{BO} \mathrm{n}^{\circ} 37$ du 11 octobre 2012.

3 Brigitte Cervoni, Fatima Chnane-Davin et Manuela Ferreira-Pinto, Entrée en matière, La méthode de français pour adolescents nouvellement arrivés, Paris, Hachette FLE, 2005 ; Elisabeth Faupin et Catherine Théron, Enseigner le FLS par les textes littéraires, Paris, Cahiers de Villes-École-Intégration du CNDP, coll. Outils pédagogiques, 2006 ; Dominique Levet, Français langue seconde, Paris, Belin, 2012.

4 Nathalie Auger, Élèves nouvellement arrivés en France - Réalités et perspectives en classe, Paris, Éditions des archives contemporaines, 2010 ; Fatima Chnane-Davin (éd.), Le français langue seconde en milieu scolaire français. Le projet CECA, Grenoble, PUG, 2011 ; Christine Hélot, Du bilinguisme en famille au plurilinguisme à l'école, Paris, L'Harmattan, 2007 ; Catherine Klein (éd.), Le français comme langue de scolarisation. Accompagner, enseigner, évaluer, se former, Futuroscope, CNDP, 2012 ; Danièle Moore et Cécile Sabatier, Une semaine en classe d'immersion française au Canada. Le projet CECA au Canada, Grenoble, PUG, 2012. 
Nous avons réalisé des enregistrements en classe d'accueil (UPE2A) ${ }^{5}$ et en classe ordinaire avec des élèves débutants intégrés, arrivés depuis moins de deux ans, en cours de français, de mathématiques et d'histoire-géographie.

Le premier résultat émanant de nos observations en classe ordinaire est l'absence totale de prise de parole des élèves EANA sans sollicitation de l'enseignant : nombre d'élèves peuvent ainsi passer des journées entières au collège sans prononcer un seul mot en classe.

Parmi les pistes existantes pour aider ces élèves à participer aux interactions en classe, nous souhaitons poser ici la question de la place des langues d'origine des élèves, autrement appelés langues maternelles ou encore langues premières, dans les classes.

Pour poser le contexte et dans un premier temps, nous proposons de présenter rapidement les modalités de scolarisation des EANA; puis nous reviendrons sur les notions de biographie langagière et de bilinguisme afin de cerner les perceptions du bilinguisme par les acteurs de l'Éducation nationale. Une deuxième partie de notre cadre théorique sera consacrée au système des interactions didactiques et à leur fonctionnement classique. L'intérêt de cette recherche réside dans l'observation de l'écart entre les interactions didactiques classiques et celles enregistrés en présence d'élèves allophones. Pour ce faire, nous analyserons des cas d'apparition de langues autres que le français en $\mathrm{DNL}^{6}$ (mathématiques et histoire-géographie) en classe d'accueil.

\section{Modalités d'accueil des élèves allophones nouvellement arrivés en France}

La scolarisation des élèves allophones a connu plusieurs décennies d'hésitations et de textes officiels imprécis. Aujourd'hui, c'est la circulaire n 2012-141 du 2 octobre 2012 relative à l'organisation de la scolarisation des élèves allophones nouvellement arrivés qui oriente les établissements dans leur mission. Les élèves doivent bénéficier d'une double inscription, dans une structure d'accueil et d'emblée dans une classe ordinaire, affirmant ainsi la nécessité d'inclure les élèves dans des classes correspondant à leur âge de manière à maintenir les possibilités d'orientation pour leur avenir. Ils doivent suivre douze heures de français au minimum ainsi que des heures d'enseignement

5 Unité pédagogique pour les élèves allophones arrivants. Pour un commentaire sur la dénomination des élèves allophones, voir Elisabeth Faupin, "Les élèves nouvellement arrivés au collège en France : prendre la parole en classe lorsque l'on débute en français. Analyse des interactions didactiques pour les élèves en immersion ", INITIO, 4, Minorités en éducation et dans le monde du travail, 2014, p. 35.

6 Nous utilisons par commodité l'appellation "discipline non linguistique » (DNL) pour les matières scolaires dont l'objectif premier n'est pas l'enseignement de la langue bien que cette dénomination ne considère pas l'aspect linguistique de chaque enseignement au collège. 
spécifiques (mais la circulaire ne précise pas le nombre d'heures à allouer). Dans les faits, les établissements que nous avons visités offrent entre 12 heures et 30 heures de cours pour les élèves allophones, le plancher de 12 heures étant le plus fréquemment observé. Les enseignants qui interviennent dans les cours de FLS sont généralement diplômés ou pourvus d'une solide expérience dans la scolarisation des élèves migrants. En revanche, parmi les quatre-vingt-dixsept enseignants d'autres disciplines consultés, seuls $10 \%$ ont déjà participé à une formation sur la scolarisation des EANA. Les professeurs accueillent donc des élèves allophones dans les classes ordinaires sans avoir été formés pour le faire, sans avoir eu l'occasion, par exemple, de s'informer sur l'évolution des définitions du bilinguisme et sur la notion de biographie langagière.

\section{Biographie langagière et bilinguisme}

Cette absence quasi totale des langues premières des élèves à l'école pose la question les pratiques langagières en situation didactique. Les acteurs du système scolaire ont pourtant conscience que parmi les élèves scolarisés en France, nombreux sont ceux qui parlent une autre langue que le français. En 1994, Christine Deprez estimait déjà " qu'en région parisienne environ un enfant sur quatre parle ou comprend une autre langue que le français ${ }^{7}$. De nombreux élèves ont donc des capacités dans deux ou plusieurs langues, autrement dit ils ont une biographie langagière riche et le plus souvent, leurs professeurs l'ignorent. La tradition monolingue, si bien installée en France, ne nous permet pas de prendre conscience comme l'écrivent Georges Lüdi et Bernard Py que « [p]lus de la moitié de l'humanité est plurilingue ou vit dans un environnement multilingue $»^{8}$. Cette notion de biographie langagière nous amène à examiner les différentes définitions du bilinguisme car bien souvent, les enseignants ne voient pas dans ces langues parlées dans les familles des élèves un atout ; elles sont plutôt considérées comme des obstacles à l'apprentissage du français. Et bien que la définition du bilinguisme ait largement évolué ces dernières décennies, nous vivons encore les conséquences de la définition établie par L. Bloomfield qui soutenait en 1935 que le bilinguisme correspond à la " connaissance de deux langues comme si elles étaient toutes deux maternelles ${ }^{9}$. Or, l'acception actuellement admise découle de la définition de François Grosjean qui, en 1984, proposait une signification plus fonctionnelle : " est bilingue la personne qui se sert de deux langues dans la vie de tous les jours et non qui possède une maîtrise semblable (et parfaite)

7 Christine Deprez, Les enfants bilingues : langues et familles, Paris, Didier, 1994, p. 15.

8 Georges Lüdi et Bernard Py, Etre bilingue, Bern, Peter Lang, 1986-2003, p. 1.

9 Leonard Bloomfield, Language, Londres, Allen \& Unvin, 1935. Le langage, Paris, Payot (trad. fr. 1970), p. 57. 
des deux langues ${ }^{10}$. Cette vision permet d'établir le plurilinguisme comme une norme dans le monde.

\section{Les perceptions du bilinguisme : interférences et interlangue}

En didactique des langues étrangères, la prise en compte du répertoire verbal des apprenants est une notion qui s'est développée récemment. La formation continue des enseignants étant bien insuffisante, peu d'entre eux ont accès à ces nouvelles orientations. On peut faire l'hypothèse que la prédominance dans la deuxième moitié du $\mathrm{XX}^{\mathrm{e}}$ siècle des théories de l'analyse contrastive influence encore les enseignants de langue en poste actuellement. Pour l'analyse contrastive, la mise en parallèle de deux langues, la langue source et la langue cible, doit permettre de prévoir toutes les difficultés rencontrées par les apprenants. "Ce qui est proche ou semblable est facile à apprendre, ce qui est différent donne lieu à un transfert négatif et donc à des fautes ${ }^{11}$ explique Martine Marquilló Larruy dans son ouvrage sur l'interprétation de l'erreur. Ce point de vue sur l'apprentissage des langues a donné naissance à la notion d'interférence selon laquelle la langue 1 est envisagée comme un obstacle à l'apprentissage de la langue 2 dans la mesure où elle risque d'induire des erreurs.

Pourtant, on sait depuis longtemps que des personnes bilingues pratiquent un "mélange des langues $»^{12}$. C'est pour analyser ce phénomène, que John Gumperz a développé la notion de " code-swiching " ${ }^{13}$ ou " alternance codique "pour qualifier l'alternance des langues.

Les critiques de l'analyse contrastive finissent par déboucher dans les années 1970 sur la notion d'interlangue, définie comme "l'ensemble des connaissances intermédiaires qu'un sujet a d'une langue seconde qu'il est en train d'apprendre $»^{14}$; la langue 1 n'est plus envisagée comme un obstacle mais comme une référence. Dans cette perspective, si l'objectif de la didactique des langues est de construire une compétence plurilingue, comme l'encourage aujourd'hui le Conseil de l'Europe, l'alternance des langues n'est plus considérée comme une absence de compétence dans la langue cible mais comme un bilinguisme en devenir. Avant les années 1960, les chercheurs sur l'apprentissage des langues avaient souvent conclu au désavantage du bilinguisme sur le plan cognitif. Depuis plusieurs années, tous s'accordent pour reconnaitre les atouts du bilinguisme.

10 François Grosjean, Le bilinguisme : vivre avec deux langues, TRANEL, 7, 1984, p. 16.

11 Martine Marquilló Larruy, Linterprétation de l'erreur, Paris, Clé International, 2003.

12 Georges Lüdi et Bernard Py, Etre bilingue, Bern, Peter Lang, 1986, 2003, p. 141.

13 John J. Gumperz, Language and social identity, Cambridge, Cambridge University Press, 1982.

14 Lüdi et Py, op. cit., 1982, p. 114. 
Parallèlement à ce consensus de la part des chercheurs, nous devons interroger la vision des enseignants sur le terrain car elle ouvre un nouveau questionnement : comment encourager les enseignants de toutes disciplines à évoluer en direction de ces représentations afin d'étayer les apprentissages des élèves allophones?

\title{
Un bilinguisme invisible
}

Notre corpus montre que malgré un fort pourcentage d'élèves concernés par le bilinguisme, les langues de première socialisation des élèves sont très peu présentes dans les classes. Ce phénomène a été étudié par Christine Hélot, qui parle pour ces élèves, de "bilinguisme invisible $»^{15}$. On peut y voir une forme de rejet de ces compétences langagières de la part de l'école car le plus souvent les enseignants n'ont pas conscience des compétences acquises par leurs élèves. Fatima Chnane-Davin a montré que ce phénomène est vu, non comme la construction de plusieurs compétences, mais comme le passage d'un monolinguisme à un autre monolinguisme :

\begin{abstract}
"L'école exige que la socialisation et la scolarisation ne se fassent qu'en français sans prendre en compte le fait qu'abandonner une langue pour aller vers une autre crée une rupture entre deux mondes linguistiques, culturels et sociaux constitutifs de l'identité de l'individu. Par conséquent, on passe d'un monolinguisme dans la langue d'origine à un monolinguisme en français. [...]

Dans cette situation d'enseignement-apprentissage, il manque, évidemment, une étape intermédiaire qui consiste en un travail de contact de la langue maternelle et de la langue seconde (Auger, 2005 ${ }^{16}$ ) pour se construire une (nouvelle) biographie langagière. L'absence de cette étape plurilingue fait que l'élève n’arrive pas à lier les connaissances linguistiques de sa langue maternelle et celles de la langue seconde ${ }^{17}$. "
\end{abstract}

En classe, l'enseignant attend des élèves allophones qu'ils entrent dans la langue française sans pouvoir ni montrer qu'ils possèdent d'autres connaissances ni s'appuyer sur ces savoirs acquis antérieurement.

\section{Analyser les irruptions de langue 1 dans les interactions didactiques}

Nous avons donc voulu observer comment se déroulent les échanges didactiques lorsqu'un élève ressent le besoin d'utiliser une langue autre que le français. Certains chercheurs, comme Simona Pekarek ${ }^{18}$, ont analysé les irruptions des langues premières en classe de langue étrangère en contexte scolaire

15 Christine Hélot, Du bilinguisme en famille au plurilinguisme à l'école, Paris, L'Harmattan, 2007, p. 12.

16 Nathalie Auger, Comparons nos langues, Montpellier, Scéren, 2005.

17 Fatima Chnane-Davin, Le français langue seconde en milieu scolaire français. Le projet CECA, Grenoble, PUG, 2011, p. 45 et p. 53.

18 Simona Pekarek, Leçon de conversation : dynamiques de l'interaction et acquisition de compétences discursives en classe de langue seconde, Fribourg, Éditions universitaires de Fribourg, 1999. 
dans le cas où l'enseignant a une langue en partage avec les élèves : les professeurs, qui cherchent souvent à éviter l'apparition de la langue 1, s'interrogent sur le déroulement des échanges en classe et plus précisément sur le moment où les élèves cessent de parler dans la langue cible pour passer en langue 1. Parallèlement il existe des recherches sur l'utilisation de deux langues par les locuteurs bilingues dans la vie ordinaire. Danièle Moore affirme par exemple que l'irruption de la langue première dans une conversation en langue 2 ne doit pas forcément être interprétée comme un manque, une lacune dans la connaissance de la langue 2 . Elle appelle "bouées transcodiques ${ }^{19}$ ces moments où les élèves de cours de langue vivante reviennent à la langue 1 : ces «bouées " fonctionnent selon l'auteure comme des balises du dysfonctionnement, autrement dit, elles permettent la focalisation sur le lieu de détresse et l'appel à l'aide, mais surtout elles permettent de maintenir le contact, elles évitent la rupture dans la communication.

Pour observer le fonctionnement des interactions, nous nous appuyons également sur une tradition d'analyse des interactions et plus particulièrement des interactions didactiques qui ont pour particularité de fonctionner dans un contexte dans lequel les positions des participants aux échanges sont asymétriques.

Ces recherches sur les interactions didactiques ont montré depuis longtemps que l'interaction classique prenait la forme d'une séquence $\operatorname{IRE}^{20}$ :

$\square$ initiative

$\square$ réponse

$\square$ évaluation

Ce schéma prend une forme ternaire : l'enseignant pose une question (initiative), un élève répond et l'enseignant valide, ou pas, la réponse apportée par l'élève. Bien que découverte il y a plusieurs décennies, cette séquence IRE est très présente dans notre corpus. Ce schéma reste donc très fréquent en contexte scolaire.

Dans les conversations en situation exolingue, l'asymétrie de la position des locuteurs peut se transformer en situation didactique si une forme de contrat est acceptée par les participants à l'échange. Jean-François De Pietro, Marinette Matthey et Bernard $\mathrm{Py}^{21}$ ont mis en lumière les « séquences potentiellement acquisitionnelles » ou SPA qui prennent la forme suivante au cours de l'échange :

19 Danièle Moore, "Bouées transcodiques en situation immersive ou comment interagir avec deux langues quand on apprend une langue étrangère à l'école ", AILE, 7, 1996, p. 95-122.

20 John McHardy Sinclair et Malcolm Coulthard, Toward an analysis of discourse: the English used by teachers and pupils. London, Oxford University Press, 1975.

21 Jean François De Pietro, Marinette Matthey et Bernard Py, "Acquisition et contrat didactique : les séquences potentiellement acquisitionnelles de la conversation exolingue ", in Dominique Weil et Huguette Fugier (éds), Actes du troisième colloque régional de linguistique, 1989, Strasbourg, Université des sciences humaines et Université Louis Pasteur, p. 99-119. 
$\square$ obstacle d'encodage

$\square$ intervention-proposition

reprise

Ces séquences interviennent dans la conversation entre un natif et un non natif, lorsqu'une forme de " contrat didactique » entre les deux participants se met en place : l'asymétrie des positions étant acceptée, le locuteur natif peut corriger ou reprendre le locuteur non natif pour l'aider à progresser. $\mathrm{Si}$ le locuteur natif accepte l'aide proposée au cours de la conversation, il peut faire de nouvelles acquisitions dans la langue cible. Ces séquences prennent également une forme ternaire : le locuteur non natif ne connaît pas un mot dans la langue 2 (obstacle d'encodage) ; le locuteur natif donne le mot dans la langue 2 (intervention-proposition) et le locuteur non natif le reprend, le répète éventuellement pour le retenir. Ces deux séquences, IRE en contexte scolaire et SPA en situation exolingue, reposent toutes deux sur un contrat didactique ; néanmoins ce contrat n'est pas comparable : en situation scolaire le contrat est imposé par l'institution alors que dans la conversation exolingue, le contrat est reconnu par les participants.

L'analyse des interactions didactiques de notre corpus révèle qu'il contient à la fois des séquences IRE et des SPA ; ces apparitions s'expliquent par le contexte particulier aux classes d'accueil des élèves allophones. Nous sommes à la croisée des deux situations décrites plus haut : une situation à la fois didactique et exolingue (réellement exolingue, ce qui n'est pas le cas des cours de langue vivante en collège dans lesquelles l'enseignant parle la langue 1 des élèves). Ce contexte donne ainsi naissance à une nouvelle séquence qui prend une forme hybride :

\section{initiative}

$\square$ obstacle d'encodage-réponse en langue 1

$\square$ réponse en langue cible

Nous sommes donc face à une séquence ternaire qui est à l'initiative de l'enseignant, schéma classique de la situation didactique ; l'élève rencontre un problème pour formuler sa réponse et utilise sa langue 1. La séquence se termine par une réponse donnée par l'enseignant en langue cible.

Pour rendre ces considérations théoriques plus concrètes, nous proposons d'observer quelques exemples d'échanges enregistrés dans les classes de collèges. Comme nous l'avons dit, les langues 1 des élèves arrivants n’apparaissant jamais en classe ordinaire, tous les exemples avancés ci-dessous sont extraits de cours d'histoire-géographie et de mathématiques en classe d'accueil, classes dans lesquelles tous les élèves sont allophones et récemment arrivés en France. Dans ce cadre, les enseignants de DNL qui interviennent auprès des publics d'UPE2A préparent leurs cours de façon autonome, sans concours du professeur de FLS. 


\title{
Analyse d'extraits du corpus : l'apparition des langues 1 en cours de DNL
}

\author{
Les cas d'acceptation
}

Extrait 1 : cours d'histoire « La fondation de Rome $»^{22}$

Dans un cours d'histoire pour les élèves UPE2A, le professeur a pour objectif de préparer les élèves à suivre rapidement les cours d'histoire-géographie dans leur classe-mère. Ce jour-là, l'enseignant commence une nouvelle leçon pour des EANA et explique le terme " fondation" :

$285 \mathrm{P}$ voilà ici vous notez s'il vous plaît // en un /// la fondation (27s) comment est-ce qu'on appelle ça en français > / vous savez> / euh par exemple la fondation / y a d'autres mots / quand : un : / une personne / arrive au monde / comment est-ce qu'on appelle ça $</ /$ quand quelqu'un arrive au monde / un bébé / Joao Joao Pedro

286 G Nascimento

287 P nacimiento on dit comme ça en espagnol et en portugais : non> c'est la naissance> / la fondation c'est la naissance / c'est un synonyme> / c'est / un nom qui veut dire la même chose

Dans cet exemple, nous sommes en situation didactique : l'enseignant prend l'initiative établissant un parallèle avec le mot "naissance " pour expliquer "fondation ». Ce faisant, il introduit la question, très intéressante, de l'utilisation du lexique dans des acceptions différentes selon les disciplines. On observe une gradation de l'étayage pour aider les élèves à trouver le terme : "fondation " / " quand une personne arrive au monde " / "un bébé ». La réponse de l'élève apparaît en langue 1 (portugais), ce qui n'est pas conforme au contrat didactique (les enseignants demandent que les élèves ne parlent pas une langue inconnue du professeur) et dans la troisième intervention, c'est l'enseignant qui donne la réponse en français "c'est la naissance». Cette séquence illustre le schéma annoncé ci-dessus : initiative, obstacle d'encodage (qui engendre ici une

22 Conventions de transcription

$\mathrm{P}$ professeur

G élève garçon

$\mathrm{F}$ élève fille

$\mathrm{Cl}$ classe

-G même locuteur qu'à l'avant dernier tour

I pause courte

(27s) pause de 27 secondes

: $\quad$ allongement de la syllabe

$>$ intonation montante

$<\quad$ intonation descendante

[ chevauchements

A accentuation 
réponse en langue 1) et réponse par l'enseignant en langue cible. Par ailleurs, on voit que cet enseignant est à l'aise avec l'irruption d'une langue étrangère, une langue autre que la langue de scolarisation, qui pourrait pourtant venir déstabiliser son statut de meneur de jeu, de détenteur du savoir : il répète un mot dans une langue étrangère, ce qui est un phénomène rarissime dans les classes.

Extrait 2 : cours de géographie « Les domaines »

Ce deuxième exemple est extrait d'un cours de géographie sur les domaines avec le même enseignant ; l'obstacle d'encodage est traité différemment par le professeur :

155 P des f- des sapins effectivement forêt de sapins / alors comment est-ce qu'on appelle ce: cet euh cet endroit / situé entre deux montagnes / dans un un paysage de montagnes / Nelson>

156 G le val

157 P la vallée / c'est ça c'est exactement ça c'est la vallée / effectivement

Du point de vue de l'élève, la situation est identique : pour répondre à la question de l'enseignant qui cherche à faire émerger le mot "vallée », il fait le pari que le terme dans sa langue sera proche du mot français et fait une tentative " le val " ( o val » en portugais, langue première de l'élève). L'enseignant reformule directement et la réponse de l'élève fait l'objet d'une évaluation positive du professeur " c'est ça c'est exactement ça c'est la vallée / effectivement " sans remarque concernant la langue de formulation. L'emploi de la langue première de l'élève n'a pas causé de rupture dans la communication grâce à l'acceptation par cet enseignant d'une langue qui ne fait pas partie du contrat didactique généralement admis au collège. Pourtant, en règle générale, les élèves intègrent très rapidement l'interdiction de l'utilisation de la langue 1 ou l'incongruité de sa présence en classe, même dans les structures d'accueil ; à plusieurs reprises dans notre corpus, les élèves manifestent leur gêne (en riant, par exemple) lorsque l'un d'entre eux utilise une langue que l'enseignant ne connaît pas. Ils développent donc des stratégies pour tenter de palier un obstacle d'encodage lorsqu'ils souhaitent répondre à une question du professeur dont voici deux exemples.

\section{Les cas d'évitement de la langue 1}

\section{Extrait 3 : utilisation d'une forme francisée}

La transcription ci-dessous correspond à un extrait de cours de géographie pour UPE2A sur les grandes métropoles avec un autre enseignant. Après avoir travaillé sur la ville de Paris, ils étudient New York. Pour ce faire, l'enseignant montre, sur Google Earth, des espaces caractéristiques de la ville : 


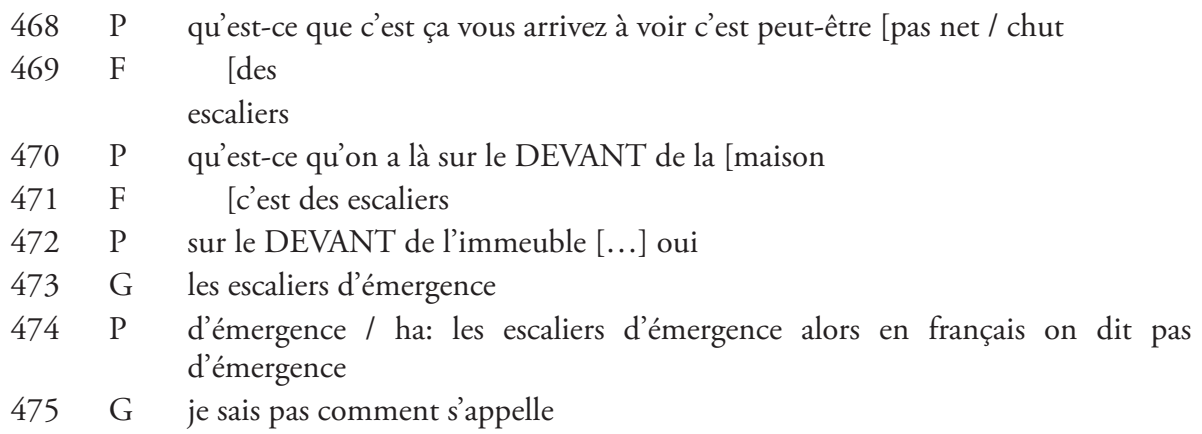

L'enseignant indique un élément sur l'image et une fille répond " des escaliers " mais le professeur ne valide pas cette intervention et reformule sa question (470). L'élève répète sa réponse mais le professeur l'ignore car il s'agit d'un cas d'auto-sélection qui crée un chevauchement. L'enseignant n'a pas donné la parole à cette élève, il n'accepte pas la réponse " c'est des escaliers " qui est d'ailleurs partielle. En 472, le professeur sélectionne un garçon dont la langue 1 est le portugais qui tente une traduction du mot " emergencia " (" urgence ») C'est pour lui un moyen, comme le montre D. Moore pour le cours de langue 2, de maintenir la communication sans la briser.

Néanmoins, le mot "émergence » est prononcé avec une intonation française (473), et l'enseignant accepte partiellement la réponse car il répète le mot erroné à trois reprises (474) afin d'amener les élèves à compléter la réponse par étayages successifs. L'apparition du mot ne représente pas une mise en difficulté de l'enseignant car la prononciation est typiquement française. Malgré l'alternance codique, la communication didactique n'est pas rompue, et, par la suite, une élève portugaise donnera le terme attendu (escalier de secours). L'échange est de forme IRE c'est-à-dire identique à un échange monolingue :

$\square$ question du professeur

$\square$ réponse mi-langue $1 /$ mi-langue 2

$\square$ évaluation négative du professeur (suivi d'une nouvelle question qui renouvelle la séquence ternaire)

L'échange se termine avec un aveu d'ignorance « je sais pas comment s'appelle » (475), qui peut ressembler à une forme d'appel de la part de l'élève mais qui est également une manière de dire "Je le sais dans ma langue ", situation qui est représentée explicitement dans l'exemple suivant enregistré durant le même cours :

\section{Extrait $4:$ dire qu'on sait}

589 P regardez / dans les villes< / au États-Uni:s / hein à New York à Chicago à Los Angeles où vous voulez / hé ben quand on regarde la ville d'en haut / on s'aperçoit que les rues> / sont / vous l'avez pas fait en mathématiques / encore ça / non / ça a pas l'air 
$590 \quad$ G je sais le mot en portugais mais en français

$591 \mathrm{P} \quad$ ah tu le sais en portugais / bon ben on va l'apprendre en français alors

592 G Quartier

593 P Répète

594 G Quartier

595 P quartier> tu dis> / non on parle de QUAdrillage

Dans cette séquence, l'élève ne tente pas une traduction pour répondre à la question de l'enseignant mais le sollicite pour montrer qu'il sait. Il veut montrer qu'il connaît la notion même si le mot français lui fait défaut ; il s'agit aussi d'une manière de poursuivre le jeu interactionnel didactique et de jouer son rôle d'élève qui répond aux questions de l'enseignant. Pourtant, il ne s'autorise pas à dire le mot en langue 1 : il a déjà intégré les codes interactionnels de l'enseignant, qui, contrairement au précédent, n'envisage pas l'apparition des langues d'origine dans son cours. Du point de vue de l'élève, cette situation est à rapprocher de la frustration dont parle Jim Cummins lorsqu'il explique que pour les élèves allophones, ne pas pourvoir " communiquer leur intelligence, leurs sentiments, leurs idées et leur humour aux enseignants et aux pairs ${ }^{23}$ peut être frustrant.

Mais l'accueil qui est fait aux langues autres que la langue de scolarisation dans les classes dépend aussi de la proximité de l'enseignant avec ces différentes langues.

\section{Le degré de xénité}

\section{Extrait 5 : une intervention en russe}

Dans un cours de mathématiques pour les EANA en classe d'accueil, un enseignant souhaite introduire le vocabulaire des formes géométriques. Dans l'exemple qui suit, le mot attendu émerge en langue 1 qui est le russe :

469 P alors je vais peut-être commencer par les plu:s simples // est-ce que quelqu'un connaît ça>

470 G Quadra

471 P alors (rire) oui mais moi j’aimerais le mot français

$472 \mathrm{Cl}$ (rires)

473 F Quatre

474 G Heu

475 F *carré

476 P Comment

477 F heu un carré

478 P un carré d'accord un carré

23 Jim Cummins, Favoriser la littératie en milieu multilingue, Secrétariat de la littératie et de la numératie, Monographie n ${ }^{\circ}$, Juin, Ontario, 2002, p. 3. 
Après la question de vocabulaire de l'enseignant, le réflexe linguistique d'un garçon de la classe est d'utiliser le mot dans sa langue 1 (470). On peut faire l'hypothèse que le rire de l'enseignant qui suit (471) est l'expression d'une gêne, car dans ce même cours d'autres occurrences d'apparitions d'interventions en langues 1 entraînent un petit rire systématique de l'enseignant. D'ailleurs, les rires des autres élèves en 472 montrent que les camarades ont déjà intégré les codes de l'école française quant au statut des langues d'origine. Dès lors, ces rires signifient à l'élève que l'usage du russe est incongru dans le contexte de la classe. Il faut signaler que ce professeur de mathématiques débute avec les UPE2A sans formation préalable. Malgré sa volonté de bien faire, il n'a probablement pas eu le loisir de réfléchir sur le rôle et le statut des langues d'origine. Dans cette séquence, même en l'absence de connaissance du russe, il était possible de prendre en compte la réponse de l'élève en s'écartant un peu de la planification pour mieux y revenir en s'appuyant sur la base latine du mot russe "quadra " pour enseigner finalement le terme français.

\section{Extrait 6 : une intervention en anglais}

C'est parfois l'enseignant qui est à l'origine de l'apparition d'une langue autre que la langue de scolarisation dans le cas par exemple où il souhaite vérifier la compréhension. Dans ce même cours de mathématiques pour UPE2A, le professeur veut expliquer la différence entre « cercle " et " disque "; pour cela, il a besoin de l'adjectif " vide» :

588 P alors le cercle / il n'y a rien dedans / d'accord c'est vide / vous comprenez> vide>

589 G Non

$590 \quad$ P Empty

591 G empty ha yes

592 P hein heu vide heu /// ma boîte est vide / d'accord vide il n'y a rien dedans // il n’y a rien à l'intérieur // par contre / ici

$593-\mathrm{G}$ ici il y a

$594 \quad \mathrm{P} \quad$ ici ce n'est pas vide

$595-\mathrm{G}$ ah ouais

596 P d'accord alors ça porte un autre nom / ça s'appelle un disque

587 G Disque

$598 \mathrm{P} \quad$ un disque / comme pour la musique

L'enseignant tente d'utiliser l'adjectif « vide » en 588, mais un élève signale son incompréhension " non ». La traduction " empty " proposée par l'enseignant engendre immédiatement l'assentiment d'un garçon en 591 « empty ha yes ». Mais on peut se demander s'il n'y a pas là un risque de laisser de côté les élèves qui ne sont pas anglophones. On voit que l'enseignant est conscient de la nécessité de ne pas en rester là et de proposer une explication de l'adjectif "vide » pour l'ensemble de la classe. Il poursuit donc en montrant une boîte vide (592) "rien à l'intérieur " avant d'introduire le lexique de la géométrie " disque » et de proposer un parallèle avec le disque de musique (598). 
Ces extraits montrent bien, que lorsque les enseignants connaissent des langues étrangères, leur attitude face à l'emploi de langues autres que le français est modifiée : l'alternance codique peut alors intervenir dans le cours à l'initiative du professeur.

Pourtant cet enseignant invoque lui-même l'anglais tandis que l'apparition du russe dans son cours provoque une gêne. Pour expliquer ce phénomène, on peut faire appel à la notion de "degré de xénité »" ${ }^{24}$, décrite par Louise Dabène en 1994, pour montrer que toutes les langues étrangères ne nous sont pas également étrangères. Pour cet enseignant, il semblerait que l'anglais comme langue d'apprentissage scolaire et langue véhiculaire soit légitime en classe, mais le russe n'y a pas sa place.

\section{Éléments de conclusion : quel traitement pour les langues pre- mières des élèves dans les cours de DNL?}

Les exemples d'apparition d'une langue étrangère en classe de mathématiques ou d'histoire-géographie pour les élèves allophones permettent de proposer quelques éléments de classification des situations étudiées. Ces apparitions peuvent être envisagées selon le point de vue de l'enseignant ou de l'élève.

Du point de vue de l'enseignant :

$\square$ La langue 1 peut être tolérée mais les réponses des élèves sont exigées en français.

$\square$ La langue 1 peut être acceptée et reprise par l'enseignant.

$\square$ La langue 1 peut être acceptée et suivie d'une reformulation par l'enseignant.

$\square$ Une langue étrangère (valorisée par notre système scolaire) à l'initiative de l'enseignant peut être convoquée, le plus souvent dans des cas d'élucidation d'un mot de vocabulaire.

Les interventions des élèves prennent des formes variées :

$\square$ Intervention en langue 1 .

$\square$ Intervention en langue 1 francisée.

$\square$ Demande d'autorisation de donner une réponse en langue 1 .

$\square$ Autocensure : cas le plus fréquent mais qui n’apparaît pas en tant que tel dans les enregistrements dans la mesure où c'est l'absence des langues 1 en classe qui prédomine.

Ces exemples soulèvent également la question du statut des langues des élèves et de l'impact de cette autocensure sur leur construction cognitive et sociale. Les enseignants ne pouvant pas connaître les langues de tous les

24 Louise Dabène, Repères sociolinguistiques pour l'enseignement des langues, Paris, Hachette FLE, 1995 , p. 35. 
élèves migrants, il serait opportun de leur proposer des formations d'éveil aux langues comme Michel Candelier en développe pour les enfants afin de leur faire percevoir les problématiques des échanges exolingues.

Pour une conclusion à visée plus large, on peut se demander dans quelles conditions est réalisée l'inclusion des élèves allophones dans des classes ordinaires lorsqu'ils viennent d'arriver en France sans aucune connaissance des langues et culture françaises. Le risque dans la situation actuelle est de voir des élèves rester silencieux durant de longs mois.

\section{Elisabeth Faupin}

CUEFLE université Nice Sophia Antipolis Elisabeth.Faupin@unice.fr

\section{Résumé}

Dans cette contribution nous analysons les marques transcodiques dans les cours de mathématiques et d'histoire-géographie en collège. La question qui se dégage ici est la manière dont les langues des élèves pourraient être utilisées par l'enseignant en classe pour véhiculer le savoir disciplinaire et comme tremplin vers la langue de scolarisation afin de faciliter le processus d'intégration scolaire et sociale.

\section{Mots-clés}

Marques transcodiques, français langue de scolarisation, intégration scolaire et sociale.

\section{Abstract}

In this paper we analyze "translinguistic markers" used in mathematic, history and geography classes in secondary schools. The main point of this study is to show how teachers could use pupils' native languages as means to convey disciplinary knowledge and as a springboard towards French language learning, facilitating school and social integration.

\section{Keywords}

Translinguistic markers, French as second language, school and social integration. 